\title{
Distribution of the quantum mechanical time-delay matrix for a chaotic cavity
}

\author{
P W Brouwer $\dagger$, K M Frahm $\ddagger$ and C W J Beenakker§ \\ $\dagger$ Lyman Laboratory of Physıcs, Harvard Unıversity, Cambridge, MA 02138, USA \\ \$Laboratorre de Physıque Quantıque, UMR 5626 du CNRS, Unıversité Paul Sabatier, \\ 31062 Toulouse Cedex 4, France \\ § Instituut-Lorentz, Leiden University, PO Box 9506, 2300 RA Leıden, The Netherlands
}

Recelved 29 December 1998

\begin{abstract}
We calculate the joint probability distribution of the Wigner-Smith time-delay matrix $Q=-1 \hbar S^{-1} \partial S / \partial \varepsilon$ and the scattering matrix $S$ for scattering from a chaotic cavity with ideal point contacts To this end we prove a conjecture by Wigner about the unitary invariance property of the distribution functional $P[S(\varepsilon)]$ of energy-dependent scattering matnces $S(\varepsilon)$ The distribution of the inverse of the eigenvalues $\tau_{1}, \quad, \tau_{N}$ of $Q$ is found to be the Laguerre ensemble from random-matrix theory The eigenvalue density $\rho(\tau)$ is computed using the method of orthogonal polynomials This general theory has applications to the thermopower, magnetoconductance, and capacitance of a quantum dot
\end{abstract}

\section{Introduction}

The time delay in a quantum mechanical scattering problem is related to the energy derivatıve of the scattering matrix $S(\varepsilon)$. Although the notion of such a relationship goes back to the historical works of Eisenbud [1] and Wigner [2], the first to propose a matrix equation linking time delay and scattering matrix was Smith [3]. He introduced the Hermitian matrix $Q=-\mathrm{i} \hbar S^{-1} \partial S / \partial \varepsilon$, now known as the Wigner-Smith time-delay matrix, and interpreted its diagonal elements as the time delay of a wavepacket incident upon the scatterer in one of $N$ scattering channels. The eigenvalues $\tau_{1}, \ldots, \tau_{N}$ of $Q$ are referred to as the 'proper delay times'.

In recent years, the issue of time delay and its connection to the scattering matrix has received considerable attention in the context of chaotic scattering [4-19]. Examples include the scattering of electromagnetic or sound waves from chaotic 'billiards' and the transport of electrons through so-called 'chaotic quantum dots' [20]. In both cases the scattering matrix $S$ and the time-delay matrix $Q$ have a sensitive and complicated dependence on microscopic parameters, such as the energy $\varepsilon$, the shape of the cavity, or (in the electronic case) the magnetic field. As a result, a statistical approach is justified, in which one addresses the statistical distribution of $S$ and $Q$ for an ensemble of chaotic cavities, rather than the precise value of these matrices for a specific cavity.

For the scattering matrix $S$, there is considerable theoretical $[8,21-24]$ and experimental [25-28] evidence that its distribution is uniform in the group of $N \times N$ unitary matrices, restricted only by fundamental symmetries, if the cavity is coupled to the outside world via ballistic point contacts. This universal distribution is Dyson's circular ensemble of randommatrix theory $[29,30]$. The fundamental problem is to calculate the distribution of the time- 
delay matrix $Q$ for this case of ideal coupling Known results include moments of tr $Q[4,12,13]$ and the entire distribution for the case $N=1[13,15]$ of a scalar scattering matrix

In a recent paper [17] we succeeded in computing the entire distribution for any dimensionality $N$ of the scattering matrix We found that the eigenvalues $\tau_{1}, \tau_{2}, \tau_{N}$ of $Q$ are statistically independent of the scattering matrix $S$, with a distribution that is most conveniently expressed in terms of the inverse delay tımes $\gamma_{n}=1 / \tau_{n}$,

$$
P\left(\gamma_{1}, \quad, \gamma_{N}\right) \propto \prod_{i<1}\left|\gamma_{l}-\gamma_{l}\right|^{\beta} \prod_{k} \gamma_{k}^{\beta N / 2} \mathrm{e}^{-\beta \tau_{\mathrm{H}} \gamma_{k} / 2}
$$

Here $\beta=1,2,4$ depending on the presence or absence of time-reversal and spin-rotation symmetry, $\tau_{\mathrm{H}}=2 \pi \hbar / \Delta$ is the Heisenberg time, and $\Delta$ is the mean level spacing of the closed cavity The function $P$ is zero if any one of the $\gamma_{n}$ 's is negative This distribution is known in random-matrix theory as the (generalized) Laguerre ensemble [30] The eigenvectors of $Q$ are not independent of $S$, except in the case $\beta=2$ of broken time-reversal symmetry For any $\beta$ the correlations are transformed away if $Q$ is replaced by the symmetrized matrix

$$
Q_{\varepsilon}=-1 \hbar S^{-1 / 2} \frac{\partial S}{\partial \varepsilon} S^{-1 / 2}
$$

which has the same eigenvalues as $Q$ The matrix of eigenvectors $U$ which diagonalizes $Q_{\varepsilon}=U$ diag $\left(\tau_{1}, \quad, \tau_{N}\right) U^{\dagger}$ is independent of $S$ and the $\tau_{n}$ 's, and uniformly distributed in the orthogonal, unitary, or symplectıc group (for $\beta=1,2$, or 4 , respectively)

In this paper we present a detaled and self contained derivation of these results, focussing on those parts of the derivation that have not been discussed or were only briefly discussed in [17] We feel that a detailed presentation of this derivation is important, because it relies on an old conjecture by Wigner [31] that had not been proven before in the literature (An unpublished proof is given in [32]) The conjecture concerns the invariance of the distribution functional $P[S(\varepsilon)]$ of the ensemble of energy-dependent scattering matrices under unitary transformations $S(\varepsilon) \rightarrow V S(\varepsilon) V^{\prime}$ (with $V, V^{\prime}$ two energy-independent unitary matrices) Our proof of Wigner's conjecture is based on the Hamiltonian approach to chaotic scattering [33], which connects the scattering matrix $S(\varepsilon)$ of the open cavity with leads to the Hamiltonian $\mathcal{H}$ of the closed cavity without leads It describes the so-called 'zero-dimensional' or 'ergodic' limit in which the time needed for ergodic exploration of the (open) cavity is much smaller than the typical escape time In [24] one of the authors (PWB) used the Hamiltonian approach to derive the unitary invariance of the distribution function $P(S)$ of the scattering matrix at a fixed energy This unitary invariance is known as Dyson's circular ensemble [29] Wigner's conjecture is the much more far reaching statement of the unitary invariance of the distribution functional $P[S(\varepsilon)]$

The outline of this paper is as follows In section 2 we formulate Wigner's conjecture in its most general form for arbitrary dimensionality $N$ of the scattering matrix (Wigner only considered the scalar case $N=1$ ) The proof follows in section 3 In section 4 we show how the distribution of the matrices $S$ and $Q$ follows from this, now proven, conjecture Then, in section 5 , the density of proper delay times is computed using the theory of orthogonal polynomials

Historically, the Wigner-Smith matrix $Q \propto \partial S / \partial \varepsilon$ was introduced to study the time evolution of a wavepacket This application is limited to the average delay time [4], essentially because knowledge of the full time dependence also requires higher derivatives of $S$ than the first Nevertheless, there exist physical observables that are enturely determined by $Q$ Some examples that have been discussed in the recent literature are the capacitance or admittance of a quantum dot [34], and the thermopower [35] In additıon to the energy derivative $\partial S / \partial \varepsilon$, one may study the derivative of $S$ with respect to an arbitrary external parameter $X$ Such a 
parameter can represent the shape of the cavity, the magnetic field, or a local impurity potential Recent examples of parametric derivatıves range from conductance derıvatıves [36] and charge pumping [37] in quantum dots to spontaneous emission [38] and photodissociation [39] in optical cavities The distribution of $\partial S / \partial X$ can be obtained by methods similar to those used for the derivation of $\partial S / \partial \varepsilon$ A brief discussion is given in section 6 We conclude in section 7

\section{Probability distribution of the scattering matrix and Wigner's conjecture}

In order to describe the energy dependence of the scattering matrix $S(\varepsilon)$, we make use of the so-called Hamiltonian approach to chaotic scattering $[8,33,40]$ In this approach, the $N \times N$ scattering matrix $S(\varepsilon)$ is expressed in terms of an $M \times M$ random Hermitian matrix $\mathcal{H}$ and an $M \times N$ coupling matrix $W$,

$$
S(\varepsilon, \mathcal{H})=1-2 \pi 1 W^{\dagger}\left(\varepsilon-\mathcal{H}+1 \pi W W^{\dagger}\right)^{-1} W
$$

The Hermitian matrix $\mathcal{H}$ models the Hamiltonian of the closed cavity, its dimension $M$ being taken to infinity at the end of the calculation The coupling matrix $W$ contains matrix elements between the scattering states in the leads and the states localized in the cavity We distinguish three symmetry classes, labelled by the parameter $\beta \quad \beta=1$ (2) in the presence (absence) of time-reversal symmetry and $\beta=4$ in the piesence of both time reversal symmetry and spin-orbit scattering The elements of $\mathcal{H}$ are real (complex, quaternion) numbers for $\beta=1$ $(2,4)$ The symmetries of $\mathcal{H}$ imply the following symmetries for $S \quad S$ is unitary symmetric $(\beta=1)$, unitary $(\beta=2)$, or unitary self dual $(\beta=4)$

For the problem of chaotic scattering one considers a statıstical ensemble of cavities, which may be obtained by, for example, varying the shape of the cavity We ask for the average of some function $f\left[S\left(\varepsilon_{1}\right), \quad, S\left(\varepsilon_{N}\right)\right]$ over the ensemble In the Hamiltonian approach, this ensemble average is represented by an integration over the matrix $\mathcal{H}$, which is taken to be a random Hermitian matrix with probability distribution $P(\mathcal{H})$,

$$
\left\langle f\left[S\left(\varepsilon_{1}\right), \quad, S\left(\varepsilon_{N}\right)\right]\right\rangle=\int \mathrm{d} \mathcal{H} P(\mathcal{H}) f\left[S\left(\varepsilon_{1}, \mathcal{H}\right), \quad, S\left(\varepsilon_{N}, \mathcal{H}\right)\right]
$$

Usually $\mathcal{H}$ is taken from the Gaussian ensembles from random-matrix theory [30],

$$
P(\mathcal{H}) \propto \exp \left(-\sum_{n=1}^{M} F\left(E_{n}\right)\right) \quad F(E)=\frac{\beta \pi^{2}}{4 M \Delta} E^{2}
$$

where $E_{1}, E_{2}, \quad, E_{M}$ are the eigenvalues of $\mathcal{H}$. The precise choice of $F(E)$ is not important in the limit $M \rightarrow \infty[5,41]$

The ensemble average defined by equation (22) can also be formulated in terms of a distribution function of the scattering matrix $S$

$$
\left\langle f\left[S\left(\varepsilon_{1}\right), \quad, S\left(\varepsilon_{n}\right)\right]\right\rangle \equiv \int \mathrm{d} S_{1} \quad \mathrm{~d} S_{n} P\left(\varepsilon_{1}, \quad, \varepsilon_{n}, S_{1}, \quad, S_{n}\right) f\left(S_{1}, \quad, S_{n}\right)
$$

Here $P\left(\varepsilon_{1}, \varepsilon_{2}, \quad, \varepsilon_{n}, S_{1}, \quad, S_{n}\right)$ is the joint probability distribution of the scattering matrix $S$ at the energies $\varepsilon_{1}, \varepsilon_{2}, \quad, \varepsilon_{N}$ The measure $\mathrm{d} S$ is the unique measure that is invariant under transformations

$$
S \rightarrow V S V^{\prime}
$$

where $V$ and $V^{\prime}$ are arbitrary unitary matrices $\left(V^{\prime}=V^{\mathrm{T}}\right.$ for $\beta=1$ and $V^{\prime}=V^{\mathrm{R}}$ for $\beta=4$ where $\mathrm{T}$ denotes the transpose and $\mathrm{R}$ the dual of a matrix)

Statıstical averages of the form (22) are completely characterized by the $N$ eigenvalues of $W^{*} W$ For the case of ballistic point contacts (or 'ideal leads'), these eigenvalues are all 
equal to $M \Delta / \pi^{2}[8,24,33]$. After application of suitable basis transformations on $S$ and $\mathcal{H}$ one therefore has $W_{\mu n}=\delta_{\mu n}(M \Delta)^{1 / 2} / \pi$ for $\mu=1, \ldots, M, n=1, \ldots, N$. In this case, the distribution $P(\varepsilon, S)$ of $S(\varepsilon)$ at one single energy is found to be particularly simple [8,24],

$$
P(\varepsilon ; S)=\text { constant. }
$$

Equation (2.6) is the starting point of the 'scattering-matrix approach' to chaotic scattering [5]. The ensemble of scattering matrices that is defined by the probability distribution (2.6) and the invariant measure $\mathrm{d} S$ is Dyson's circular ensemble from random-matrix theory [29]. Depending on the symmetry class, we distinguish the circular orthogonal, unitary, and symplectic ensembles (COE, CUE, CSE) for $\beta=1,2$, and 4 respectively.

The distribution function (2.6) is invariant under the transformation (2.5). Wigner [31] conjectured that the unitary invariance extends to the distribution functional $P[S(\varepsilon)]$, i.e. to the whole energy-dependent $S$-matrix ensemble. In other words, Wigner's conjecture is that (for any $n$ ) $P\left(\varepsilon_{1}, \ldots, \varepsilon_{n} ; S_{1}, \ldots, S_{n}\right)$ is invariant under a simultaneous transformation

$$
S_{j} \rightarrow V S_{j} V^{\prime} \quad j=1, \ldots, n,
$$

where $V$ and $V^{\prime}$ are arbitrary unitary matrices $\left(V^{\prime}=V^{\mathrm{T}}, V^{\mathrm{R}}\right.$ for $\left.\beta=1,4\right)$.

\section{Proof of Wigner's conjecture}

Our proof of Wigner's conjecture consists of two parts. We first present an alternative randommatrix approach for the energy dependence of the scattering matrix [16], and then show that in this approach the invariance property (2.7) is manifest.

In the alternative random-matrix approach, the role of the $M \times M$ matrix $\mathcal{H}$ is taken over by an $M \times M$ unitary matrix $U$,

$$
S(\varepsilon)=U_{11}-U_{12}\left(\mathrm{e}^{-2 \pi i \varepsilon / M \Delta}+U_{22}\right)^{-1} U_{21} .
$$

Here the matrices $U_{11}, U_{12}, U_{21}$, and $U_{22}$ denote four subblocks of $U$, of size $N \times N$, $N \times(M-N),(M-N) \times N$, and $(M-N) \times(M-N)$, respectively,

$$
U=\left(\begin{array}{ll}
U_{11} & U_{12} \\
U_{21} & U_{22}
\end{array}\right){ }_{3 M-N} N
$$

The energy dependence of $S$ enters through the phase factor $\exp (-2 \pi \mathrm{i} \varepsilon / M \Delta)$. The matrix $U$ is distributed according to the appropriate circular ensemble: $U$ is distributed according to the circular ensemble, COE (CUE, CSE) for $\beta=1(2,4)$.

We now show that equation (3.1) with $M \gg N$ is equivalent to the Hamiltonian approach (2.1). Equivalence of the circular ensemble and the Hamiltonian approach at energy $\varepsilon=0$ was proven in [24]. This allows us to write the $M \times M$ unitary matrix $U$ in terms of an $M \times M$ Hermitian matrix $\mathcal{H}$ and an $M \times M$ coupling matrix $W$,

$$
U=1+2 \pi \mathrm{i} W^{\dagger}\left(\mathcal{H}-\mathrm{i} \pi W W^{\dagger}\right)^{-1} W .
$$

The matrix $\mathcal{H}$ is distributed according to the Lorentzian ensemble [24], which in the large$M$ limit is equivalent to the Gaussian ensemble (2.3). (The Lorentzian ensemble has $\left.F(E)=\frac{1}{2}(\beta M+2-\beta) \ln \left(M^{2} \Delta^{2}+\pi^{2} E^{2}\right)\right)$.

The coupling matrix $W$ is a square matrix with elements $W_{\mu n}=\pi^{-1} \delta_{\mu n}(M \Delta)^{1 / 2}$. We separate $W$ into two rectangular blocks $W_{1}$ and $W_{2}$, of size $M \times N$ and $M \times(M-N)$, respectively,

$$
W=\left(W_{1}, W_{2}\right)
$$


and substitute equation (3.3) into equation (3.1). The result is

$$
S(\varepsilon)=1-2 \pi \mathrm{i} W_{1}^{\dagger}\left(\varepsilon-\mathcal{H}-\delta \mathcal{H}+\mathrm{i} \pi W_{1} W_{1}^{\dagger}\right)^{-1} W_{1}
$$

where the $M \times M$ matrix $\delta \mathcal{H}$ is defined as

$$
\delta \mathcal{H}=\left(\begin{array}{cc}
\varepsilon & 0 \\
0 & \varepsilon-(M \Delta / \pi) \tan (\pi \varepsilon / M \Delta)
\end{array}\right) \begin{aligned}
& \} N \\
& \} M-N
\end{aligned} .
$$

Apart from the term $\delta \mathcal{H}$, equation (3.5) is the same as equation (2.1) in the Hamiltonian approach. The extra term $\delta \mathcal{H}$ is irrelevant in the limit $M \rightarrow \infty$ at fixed $N$, because the diagonal matrix $\delta \mathcal{H}$ contains only a finite number of matrix elements that are of order $\varepsilon$, the others being of order $\varepsilon^{2} / M \Delta$. (One easily verifies that such a perturbation does not change eigenvalues and eigenvectors of $\mathcal{H}$ in the limit $M \rightarrow \infty$ ). This proves the equivalence of the alternative random-matrix approach (3.1) and the Hamiltonian approach (2.1).

It remains to derive the unitary invariance of $P[S(\varepsilon)]$ from equation (3.1). Since the matrix $U$ is chosen from the circular ensemble, its distribution is invariant under a mapping $U \rightarrow U^{\prime} U U^{\prime \prime}$, where $U^{\prime}$ and $U^{\prime \prime}$ are arbitrary $M \times M$ unitary matrices $\left(U^{\prime \prime} U^{\prime *}=1\right.$ if $\left.\beta=1,4\right)$. We now choose

$$
U^{\prime}=\left(\begin{array}{ll}
V & 0 \\
0 & 1
\end{array}\right) \begin{aligned}
& \} N \\
& \} M-N
\end{aligned} \quad U^{\prime \prime}=\left(\begin{array}{cc}
V^{\prime} & 0 \\
0 & 1
\end{array}\right) \begin{aligned}
& \} N \\
& \} M-N
\end{aligned}
$$

where $V$ and $V^{\prime}$ are arbitrary unitary $N \times N$ matrices $\left(V^{*} V^{\prime}=1\right.$ if $\left.\beta=1,4\right)$. Invariance of $P(U)$ under the transformation $U \rightarrow U^{\prime} U U^{\prime \prime}$ with the choice (3.7) implies, in view of equation (3.1), invariance of $P[S(\varepsilon)]$ under the transformation

$$
S(\varepsilon) \rightarrow V S(\varepsilon) V^{\prime}
$$

which is Wigner's conjecture.

A central role in our proof of Wigner's conjecture is played by the random-matrix model (3.1), which expresses the energy-dependent $N \times N$ scattering matrix $S(\varepsilon)$ in terms of an energy-independent $M \times M$ random unitary matrix $U$. This model was first proposed in [16], following a more physical reasoning than the formal derivation given above. It is insightful to briefly repeat the reasoning of [16]. We consider the scattering matrix $S$ at energy $\varepsilon=0$ and then shift the energy by an amount $\varepsilon$. Equivalently, we can replace the energy shift $\varepsilon$ by a uniform decrease $\delta V=-\varepsilon / e$ of the potential $V$ in the quantum dot. Since the quantum dot is chaotic, we may localize $\delta V$ in a closed lead (a stub), see figure 1 . The stub contains $M-N \gg N$ modes to ensure that it faithfully represents a spatially homogeneous potential drop $\delta V$. The scattering properties of the system consisting of the dot, the $N$-mode lead, and the $(M-N)$-mode stub are described by the $(M-N)$-dimensional reflection matrix $r_{\mathrm{s}}$ of the stub and the $M$-dimensional scattering matrix $U$ of the cavity at energy $\varepsilon=0$, with the stub

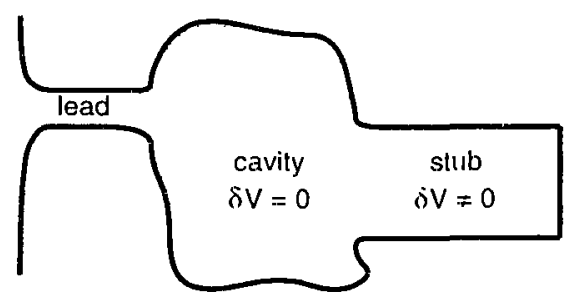

Figure 1. Physical motivation of equation (3.1), explained in the text. 
replaced by an open lead. The advantage of localizing the potential shift $\delta V$ inside the stub is that the energy dependence of $r_{\mathrm{s}}$ is very simple,

$$
r_{\mathrm{s}}(\varepsilon)=-\exp (-2 \pi \mathrm{i} \varepsilon / M \Delta) .
$$

The matrix $U$ is energy independent by construction. It is taken from the circular ensemble, because scattering from the dot is chaotic. (The matrix $r_{\mathrm{s}}$ is not random.) Expressing the scattering matrix $S(\varepsilon)$ in terms of $U$ and $r_{s}(\varepsilon)$ then yields equation (3.1).

\section{Distribution of the time-delay matrix}

In this section we combine Wigner's conjecture for the invariance properties of the scattering matrix ensemble and the Hamiltonian approach to chaotic scattering, to compute the joint distribution $P\left(S, Q_{\varepsilon}\right)$ of the scattering matrix $S(\varepsilon)$ and its symmetrized energy derivative

$$
Q_{\varepsilon}=-\mathrm{i} \hbar S^{-1 / 2} \frac{\partial S}{\partial \varepsilon} S^{-1 / 2}
$$

The matrix $Q_{\varepsilon}$ is a real symmetric (Hermitian, quaternion self-dual) matrix for $\beta=1$ (2, 4). The joint distribution $P\left(S, Q_{\varepsilon}\right)$ of $S$ and $Q_{\varepsilon}$ is defined through a relation similar to equations (2.2) and (2.4),

$$
\left\langle f\left[S(\varepsilon), Q_{\varepsilon}(\varepsilon)\right]\right\rangle=\int \mathrm{d} \mathcal{H} P(\mathcal{H}) f\left[S(\varepsilon, \mathcal{H}), Q_{\varepsilon}(\varepsilon, \mathcal{H})\right] \equiv \int \mathrm{d} S \mathrm{~d} Q_{\varepsilon} f\left(S, Q_{\varepsilon}\right) P\left(S, Q_{\varepsilon}\right)
$$

where $f\left(S, Q_{\varepsilon}\right)$ is an arbitrary function of $S$ and $Q_{\varepsilon}$.

Application of Wigner's conjecture is the key to the calculation of $P\left(S, Q_{\varepsilon}\right)$. To see this, we first consider the distribution function $P\left(\varepsilon_{2}-\varepsilon_{1} ; S_{1}, S_{2}\right)$ of the scattering matrix $S(\varepsilon)$ at the energies $\varepsilon_{1}$ and $\varepsilon_{2}$, for which the invariance property (2.7) implies

$$
P\left(\varepsilon_{2}-\varepsilon_{1} ; S_{1}, S_{2}\right)=P\left(\varepsilon_{2}-\varepsilon_{1} ; V S_{1} V^{\prime}, V S_{2} V^{\prime}\right)=P\left(\varepsilon_{2}-\varepsilon_{1} ;-1,-S_{1}^{-1 / 2} S_{2} S_{1}^{-1 / 2}\right) .
$$

For the second equality we have chosen $V=V^{\prime}=\mathrm{i} S_{1}^{-1 / 2}$. In the limit $\varepsilon_{2} \rightarrow \varepsilon_{1}=\varepsilon$, one finds from equation (4.3) that the joint distribution $P\left(S, Q_{\varepsilon}\right)$ of the scattering matrix $S(\varepsilon)$ and the symmetrized time-delay matrix $Q_{\varepsilon}$ does not depend on the scattering matrix $S$,

$$
P\left(S, Q_{\varepsilon}\right)=P\left(-1, Q_{\varepsilon}\right) .
$$

Hence, to find $P\left(S, Q_{\varepsilon}\right)$ it is sufficient to calculate the integral (4.2) for a function $f\left(S, Q_{\varepsilon}\right)$ of the form

$$
f\left(S, Q_{\varepsilon}\right)=\delta(S,-1) f\left(Q_{\varepsilon}\right)
$$

where $f\left(Q_{\varepsilon}\right)$ is an arbitrary function of $Q_{\varepsilon}$ and $\delta(S,-1)$ is the delta function at $S=-1$ on the manifold of unitary symmetric (unitary, unitary self-dual) matrices for $\beta=1(2,4)$.

We now turn to the evaluation of the integral (4.2), with $f$ of the form (4.5), using the Hamiltonian approach. We note that (2.1) can be rewritten as

$$
S=-1+\frac{2}{1+\mathrm{i} K} \quad K=\pi W^{\dagger} \frac{1}{\varepsilon-\mathcal{H}} W .
$$

The energy derivative of $S$ is given by

$$
\frac{\partial S}{\partial \varepsilon}=2 \pi \mathrm{i} \frac{1}{1+\mathrm{i} K} W^{\dagger} \frac{1}{(\varepsilon-\mathcal{H})^{2}} W \frac{1}{1+\mathrm{i} K} .
$$


We decompose the Hermitian matrix $\mathcal{H}$ in equation (42) into its eigenvalues and eigenvectors $\mathcal{H}=\psi E \psi^{*}$, where $E$ is the diagonal matrix containing the eigenvalues $E_{\rho}$ of $\mathcal{H}$ as entries and $\psi$ is the orthogonal (unitary, symplectic) matrix of the eigenvectors of $\mathcal{H}$ for $\beta=1(2,4)$,

$$
\begin{aligned}
& \left\langle f\left(S, Q_{\varepsilon}\right)\right\rangle=\int \mathrm{d} \psi \int \mathrm{d} E_{1} \quad \mathrm{~d} E_{M} J_{M}(E) P(E) f\left[S\left(\varepsilon, \psi E \psi^{\star}\right), Q_{\varepsilon}\left(\varepsilon, \psi E \psi^{\perp}\right)\right] \\
& J_{M}(E)=\prod_{\rho<\sigma}^{M}\left|E_{\rho}-E_{\sigma}\right|^{\beta}
\end{aligned}
$$

Here $\mathrm{d} \psi$ is the invariant measure on the orthogonal (unitary, symplectic) group and $J_{M}(E)$ is the Jacobian for the variable transformation $\mathcal{H} \rightarrow \psi, E$ [30]

We now make the special choice (45) for $f\left(S, Q_{\varepsilon}\right)$ In teims of the matrices $\psi$ and $E$, the matrix $K$ from equation (4 6) reads

$$
K_{m n}=\frac{M \Delta}{\pi} \sum_{\rho=1}^{M} \frac{\psi_{m \rho} \psi_{n \rho}^{*}}{\varepsilon-E_{\rho}}
$$

Inspecting (4 6), we see that the limit $S \rightarrow-1$ corresponds to the case when all $N$ eigenvalues of $K$ diverge or, equivalently, when at least $N$ out of $M$ eigenvalues $E_{\rho}$ of $\mathcal{H}$ tend to the energy $\varepsilon$ Let us label these eigenvalues $E_{1}, \quad, E_{N}, 1$ e $\left|E_{\rho}-\varepsilon\right| \rightarrow 0$ in the limit $S \rightarrow-1$ for $\rho=1, \quad, N$ In this limit, the sum in (4 9) can be restricted to the first $N$ terms and neither $S$ nor $\partial S / \partial \varepsilon$ depends on the eigenvalues $E_{\rho}$ or on the matrix elements $\psi_{m \rho}$ with $m>N$ or $\rho>N$ Therefore we can perform the integration with respect to the latter, resulting in

$$
\begin{aligned}
\left\langle\delta(S,-1) f\left(Q_{\varepsilon}\right)\right\rangle & =\int \mathrm{d} Q_{\varepsilon} f\left(Q_{\varepsilon}\right) P\left(-1, Q_{\varepsilon}\right) \\
& =\int \mathrm{d} \Psi P(\Psi) \int \mathrm{d} E_{1} \quad \mathrm{~d} E_{N} P\left(E_{1}, \quad, E_{N}\right) \delta(S,-1) f\left(Q_{\varepsilon}\right)
\end{aligned}
$$

where $\Psi$ is the $N \times N$ matrix containing the rescaled eigenvector elements $\psi_{m \rho} M^{1 / 2}$ for $1 \leqslant m, \rho \leqslant N$, the integration measure $\mathrm{d} \Psi=\prod_{m \rho=1}^{N} \mathrm{~d} \Psi_{m \rho}, P(\Psi)$ is the distribution of $\Psi$ after integration over the remaining matrix elements of $\psi$, and $P\left(E_{1}, \quad, E_{N}\right)$ is the distribution of the eigenvalues $E_{1}, \quad, E_{N}$ after integration over the remaining eigenvalues $E_{\rho}$, $\rho>N$ Near $S=-1$ we have from equations (4 6), (4 7), and (4 9),

$$
\begin{aligned}
& S=-1+(2 \pi 1 / \Delta) \Psi^{\dagger-1} \operatorname{diag}\left(E_{1}-\varepsilon, \quad, E_{N}-\varepsilon\right) \Psi^{-1} \\
& Q_{\varepsilon}=\tau_{\mathrm{H}} \Psi^{-1} \Psi^{-1}
\end{aligned}
$$

where $\tau_{\mathrm{H}}=2 \pi \hbar / \Delta$ is the Heisenberg time In the limit $M \gg N$, the matrix elements of $\Psi$ are Gaussian distributed with zero mean and unit variance [30],

$$
P(\Psi) \propto \exp \left[-(\beta / 2) \operatorname{tr} \Psi \Psi^{\dagger}\right]
$$

Because of the delta function $\delta(S,-1)$, it is sufficient to know $P\left(E_{1}, \quad, E_{N}\right)$ for $\left|E_{\rho}-\varepsilon\right| \rightarrow 0$, $\rho=1, \quad, N$ In this case, $P\left(E_{\mathrm{l}}, \quad, E_{N}\right)$ is entirely determined by the Jacobian $J_{N}\left(E_{1}, \quad, E_{N}\right)$, up to a numerical factor which results from the integration over the remaining $M-N$ elgenvalues of $\mathcal{H}$,

$$
P\left(E_{1}, \quad, E_{N}\right) \propto \prod_{\rho<\sigma}^{N}\left|E_{\rho}-E_{\sigma}\right|^{\beta}
$$

We have now succeeded in transforming the integial (42) over an $M \times M$ matrix $\mathcal{H}$ to the integral (410), which is formulated in terms of $N \times N$ matrices only At this point, it is convenient to rewrite the integration over the $N$ eigenvalues $E_{1}, \quad, E_{N}$ as a matrix integration 
to avoid the singularity of $P\left(E_{1}, \ldots, E_{N}\right)$ at $E_{1}=\ldots=E_{N}=\varepsilon$. To this end we exploit the fact that the distribution of $\Psi$ is invariant with respect to a transformation of the type $\Psi \rightarrow O \Psi, O$ being an orthogonal (unitary, symplectic) matrix for $\beta=1(2,4)$. We apply this transformation for arbitrary $O$ and average over $O$ with respect to the invariant measure $\mathrm{d} O$. Then, we substitute the Hermitian $N \times N$-matrix $H \equiv O^{\dagger} \operatorname{diag}\left(E_{1}, \ldots, E_{N}\right) O-\varepsilon$ and the corresponding Jacobian exactly cancels the singular level-repulsion factor of $P\left(E_{1}, \ldots, E_{N}\right)$,

$$
\begin{aligned}
\left\langle\delta(S,-1) f\left(Q_{\varepsilon}\right)\right\rangle & \propto \int \mathrm{d} \Psi P(\Psi) \int \mathrm{d} H \delta(S,-1) f\left(Q_{\varepsilon}\right) \\
& \propto \int \mathrm{d} \Psi P(\Psi) \int \mathrm{d} H \delta\left(\Psi^{\dagger-1} H \Psi^{-1}\right) f\left[\tau_{\mathrm{H}} \Psi^{\dagger-1} \Psi^{-1}\right] .
\end{aligned}
$$

(The proportionality sign indicates that we have omitted a normalization constant.) Three more variable transformations are needed to calculate the integral (4.15). First, we replace the integration variable $H$ by $H^{\prime}=\Psi^{\dagger^{-1}} H \Psi^{-1}$. The Jacobian $\operatorname{det}\left(\Psi \Psi^{\dagger}\right)^{(N \beta+2-\beta) / 2}$ of this transformation can be derived using the singular value decomposition of $\Psi$ [42]. The integral (4.15) becomes

$$
\begin{gathered}
\left\langle\delta(S,-1) f\left(Q_{\varepsilon}\right)\right\rangle \propto \int \mathrm{d} \Psi P(\Psi) \int \mathrm{d} H^{\prime} \delta\left(H^{\prime}\right) \operatorname{det}\left(\Psi \Psi^{\dagger}\right)^{(N \beta+2-\beta) / 2} f\left[\tau_{\mathrm{H}} \Psi^{\dagger-1} \Psi^{-1}\right] \\
=\int \mathrm{d} \Psi \exp \left[-(\beta / 2) \operatorname{tr} \Psi \Psi^{\dagger}\right] \operatorname{det}\left(\Psi \Psi^{\dagger}\right)^{(N \beta+2-\beta) / 2} f\left[\tau_{\mathrm{H}} \Psi^{\dagger-1} \Psi^{-1}\right] .
\end{gathered}
$$

Next, we note that the integrand depends on the Hermitian matrix $\Gamma \equiv \Psi \Psi^{\dagger} / \tau_{\mathrm{H}}$ only, and replace the integration variable $\Psi$ by $\Gamma=\Psi \Psi^{\dagger} / \tau_{\mathrm{H}}$. The Jacobian of this transformation $[43,44]$ provides an extra factor $\alpha \operatorname{det}(\Gamma)^{\beta / 2-1} \theta(\Gamma)$, where $\theta(\Gamma)=1$ if all eigenvalues of $\Gamma$ are positive and $\theta(\Gamma)=0$ otherwise. Finally, we replace $\Gamma$ by its inverse $Q=\Gamma^{-1}$. Since this variable transformation has a Jacobian $\operatorname{det}(Q)^{\beta N+2-\beta}$, we arrive at

$$
\left\langle\delta(S,-1) f\left(Q_{\varepsilon}\right)\right\rangle \propto \int \mathrm{d} Q \theta(Q) \operatorname{det}(Q)^{-3 \beta N / 2-2+\beta} \exp \left[-\beta \tau_{\mathrm{H}} \operatorname{tr} Q^{-1} / 2\right] f(Q) .
$$

Using equations (4.4) and (4.10) we thus find the joint probability distribution $P\left(S, Q_{\varepsilon}\right)$ of the scattering matrix $S$ and the symmetrized time-delay matrix $Q_{\varepsilon}$,

$$
P\left(S, Q_{\varepsilon}\right)=P\left(-1, Q_{\varepsilon}\right)=\theta\left(Q_{\varepsilon}\right) \operatorname{det}\left(Q_{\varepsilon}\right)^{-3 \beta N / 2-2+\beta} \exp \left[-\beta \tau_{\mathrm{H}} \operatorname{tr} Q_{\varepsilon}^{-1} / 2\right] \text {. }
$$

The corresponding distribution of the eigenvalues $\tau_{1}, \ldots, \tau_{N}$ of $Q_{\varepsilon}$, the proper delay times, then reads

$$
P\left(\tau_{1}, \ldots, \tau_{N}\right) \propto \prod_{n<m}\left|\tau_{n}-\tau_{m}\right|^{\beta} \prod_{n} \theta\left(\tau_{n}\right) \tau_{n}^{-3 \beta N / 2-2+\beta} \exp \left[-\beta \tau_{\mathrm{H}} /\left(2 \tau_{n}\right)\right]
$$

Alternatively, the distribution of the rates $\gamma_{j}=1 / \tau_{j}$ is given by

$$
P\left(\gamma_{1}, \ldots, \gamma_{N}\right) \propto \prod_{n<m}\left|\gamma_{n}-\gamma_{m}\right|^{\beta} \prod_{n} \theta\left(\gamma_{n}\right) \gamma_{n}^{\beta N / 2} \exp \left[-\beta \tau_{\mathrm{H}} \gamma_{n} / 2\right]
$$

This distribution is known in random-matrix theory as the generalized Laguerre ensemble [30]. The eigenvectors of $Q_{\varepsilon}$ are uniformly distributed according to the invariant measure of the orthogonal (unitary, symplectic) group.

\section{Density of proper delay times}

The distributions (4.19) and (4.20) have a form which allows one to apply the standard technique of orthogonal polynomials [30] to determine the density or the correlation functions of the eigenvalues of $Q_{\varepsilon}$ (or $\Gamma=Q_{\varepsilon}^{-1}$ ). The $N$-dependent exponent is somewhat unusual and implies 
that the set of orthogonal polynomials is different for each value of $N$. Such ensembles have been studied in mathematical physics $[45,46]$.

We restrict ourselves to the simplest case $\beta=2$ of broken time-reversal symmetry. For a simplified notation, we use the dimensionless escape rates $x_{n} \equiv \gamma_{n} \tau_{\mathrm{H}}=\tau_{\mathrm{H}} / \tau_{n}$ which are distributed according to

$$
p\left(x_{1}, \ldots, x_{N}\right) \propto \prod_{n<m}\left(x_{n}-x_{m}\right)^{2} \prod_{n} w_{N}\left(x_{n}\right) \quad w_{N}(x)=x^{N} \mathrm{e}^{-x} .
$$

The generalized Laguerre polynomials $L_{n}^{(N)}(x)$ are orthogonal with respect to the weight function $w_{N}(x)$. The method of ot thogonal polynomials relates the correlation functions of the $x_{n}$ 's to these polynomials. Here we consider only the density

$$
\rho(x)=\int_{0}^{\infty} \mathrm{d} x_{1} \ldots \mathrm{d} x_{N} p\left(x_{1}, \ldots, x_{N}\right) \sum_{n=1}^{N} \delta\left(x-x_{n}\right)
$$

which is given by the series

$$
\rho(x)=\sum_{n=0}^{N-1} \frac{L_{n}^{(N)}(x)^{2} w_{N}(x) n !}{(N+n) !} .
$$

Using the recurrence relation [47]

$$
(n+1) L_{n+1}^{(N)}(x)=(2 n+N+1-x) L_{n}^{(N)}(x)-(n+N) L_{n-1}^{(N)}(x)
$$

with $L_{0}^{(N)}(x)=1$ and $L_{1}^{(N)}(x)=N+1-x$, it is possible to evaluate equation (5.3) efficiently for small $N$. For large $N$, one can use an asymptotic expansion of the Laguerre polynomials to find the closed expression

$$
\rho(x)=\frac{1}{2 \pi x} \sqrt{-N^{2}+6 N x-x^{2}} .
$$

The corresponding density for the proper delay times $\tau=\tau_{\mathrm{H}} / x$ thus reads

$$
\rho(\tau)=\frac{N}{2 \pi \tau^{2}} \sqrt{\left(\tau_{+}-\tau\right)\left(\tau-\tau_{-}\right)} \quad \tau_{ \pm}=\frac{\tau_{\mathrm{H}}}{N}(3 \pm \sqrt{8}) .
$$

The limit $N \rightarrow \infty$ is rapidly approached for $N \gtrsim 3$, see figure 2 .

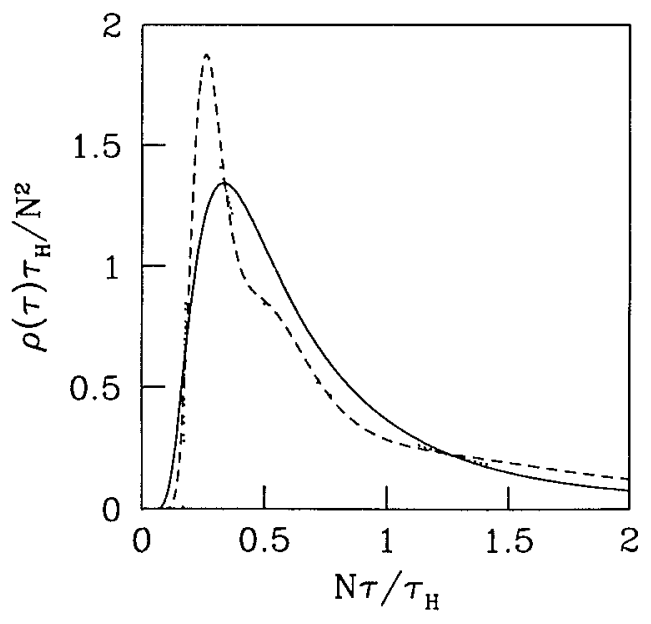

Figure 2. Density of proper delay times Shown are the densities for $N=1$ (full line), $N=3$ (dashed line), both computed from equation (53), and the limit $N \rightarrow \infty$ (dotted line) given by equation (5 6) 


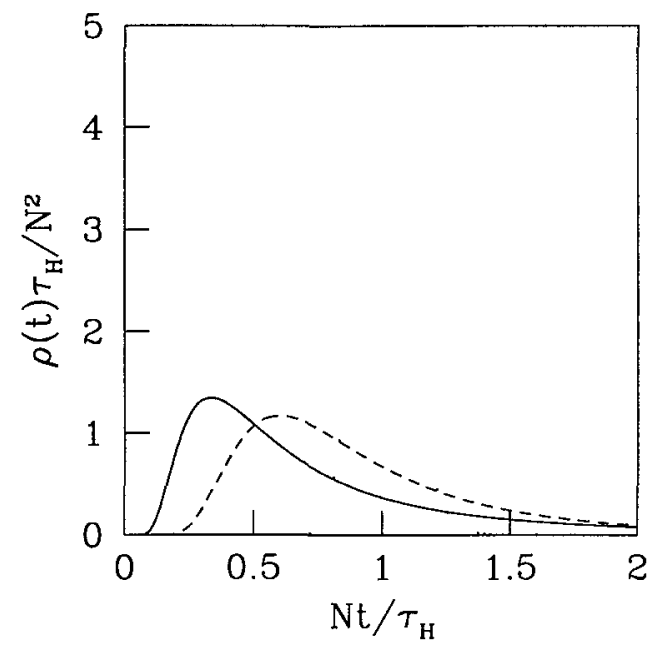

Figure 3. Density of partial delay t1mes, from [13] Shown are the densities for $N=1$ (full line), $N=3$ (dashed line) and $N=100$ (dotted line) The limit $N \gg 1$ corresponds to a Gaussian with mean 1 and width $1 / \sqrt{N}$

It is instructive to compare the density of the proper delay times $\tau_{n}$ with the density of partial delay times studied by Fyodorov, Sommers, and Savin $[4,13]$. The partial delay times $t_{n}$ are defined in terms of the eigenvalues $\mathrm{e}^{1 \phi_{n}}$ of the scattering matrix $S$,

$$
t_{n}=\hbar \frac{\partial \phi_{n}}{\partial \varepsilon}
$$

Compared to the definition of proper delay tımes as eigenvalues of the Wigner-Smith matrix, we see that for the partial delay times the order of the operations of energy derivative and diagonalization is reversed. This order is irrelevant for the sum $\sum_{n} \tau_{n}=\sum_{n} t_{n}$, corresponding to the first moment of the density, but higher moments are different (unless $N=1$ ). The density of partial delay times was obtaned in $[4,13]$ by the supersymmetric approach,

$$
\rho(t)=\frac{1}{(N-1) \tau_{\mathrm{H}}}\left(\frac{\tau_{\mathrm{H}}}{t}\right)^{N+2} \mathrm{e}^{-\tau_{\mathrm{H}} / t} .
$$

Notice that this density corresponds to the contribution from the first Laguerre polynomial in the summation (5.3). For $N \geqslant 2$, there are also contributions from higher-order Laguerre polynomials and therefore the two densities do not coincide. In figure 3, the density of the partial delay times is shown for $N=1,3,100$. For $N \gg 1$, equation (5.8) becomes a Gaussian of mean 1 and width $1 / \sqrt{N}$. The qualitative difference between $\rho(\tau)$ and $\rho(t)$ is due to the absence of level repulsion for the partial delay times. Unlike for the proper delay times, we do not know of a physical quantity that is determined by the partial delay tımes.

\section{General parametric derivatives}

So far we have restricted our discussion to the energy derivative of the scattering matrix. It is also of interest to know the derivatives with respect to external parameters. Examples of such parameters are a magnetic field, the shape of the cavity, or a local potential. In this section we present and prove an extension of Wigner's conjecture to the ensemble of scattering matrices $S(\varepsilon, X)$ that depend both on energy and on an external parameter $X$, and then use it to compute 
the distribution of the symmetrized derivative

$$
Q_{X}=-\mathrm{i} S^{-1 / 2} \frac{\partial S}{\partial X} S^{-1 / 2}
$$

In the Hamiltonian approach, the parameter dependence of the scattering matrix is modelled through equation (2.1) with a parameter-dependent random Hermitian matrix $\mathcal{H}(X)$,

$$
S(\varepsilon, X ; \mathcal{H})=1-2 \pi \mathrm{i} W^{\dagger}\left[\varepsilon-\mathcal{H}(X)+\mathrm{i} \pi W W^{\dagger}\right]^{-1} W \quad \mathcal{H}(X)=\mathcal{H}+X \mathcal{H}^{\prime}
$$

The matrix $\mathcal{H}^{\prime}$ is a random Hermitian, Gaussian distributed, symmetric (antisymmetric) matrix if $X$ denotes a shape change (magnetic field), or $\mathcal{H}_{i j}^{\prime}=\delta_{i r} \delta_{j r}$ if $X$ denotes the local potential at site $r$. (In random-matrix theory, the site $r$ is represented by an index $1 \leqslant r \leqslant M$, see e.g. [38]). As we argue below, the invariance property (2.7), which was conjectured by Wigner for the ensemble of energy-dependent scattering matrices, is also valid for the more general ensemble of parameter- and energy-dependent scattering matrices $S(\varepsilon, X)$. That is to say, the distribution functional $P[S(\varepsilon, X)]$ of the ensemble of matrix-valued functions $S(\varepsilon, X)$ is invariant under transformations

$$
S(\varepsilon, X) \rightarrow V S(\varepsilon, X) V^{\prime}
$$

where $V$ and $V^{\prime}$ are arbitrary unitary matrices $\left(V^{*} V^{\prime}=1\right.$ if $\left.\beta=1,4\right)$. The proof of this invariance property follows similar lines to that in section 3: First, one shows that the Hamiltonian approach (6.2) for the ensemble of parameter-dependent scattering matrices is equivalent to a formulation [48] in terms of unitary matrices of a form similar to equation (3.1),

$$
S(\varepsilon, X)=U_{11}+U_{12}\left[\exp \left[-2 \pi \mathrm{i}\left(\varepsilon-X \mathcal{H}^{\prime}\right) / M \Delta\right]-U_{22}\right]^{-1} U_{21} .
$$

Second, one verifies that in the formulation (6.4) the invariance property (6.3) is manifest, completing the proof.

Using the invariance property (6.3), we can now compute the distribution of $Q_{X}$. For simplicity, we restrict ourselves to the case that the matrices $\mathcal{H}$ and $\mathcal{H}^{\prime}$ have the same, Gaussian, distribution. As in the case of the energy derivative of the scattering matrix, see section 4 , it is sufficient to consider the special point $S=-1$. From equations (4.6) and (6.2) we find that at $S=-1$

$$
\begin{aligned}
& Q_{X}=\Psi^{\dagger-1} H^{\prime} \Psi^{-1} \quad P\left(H^{\prime}\right) \propto \exp \left(-\beta \operatorname{tr} H^{2} / 16\right) \\
& H_{m n}^{\prime}=-\left(\tau_{\mathrm{H}} / \hbar\right) M^{-1 / 2} \sum_{\mu, \nu} \psi_{m \mu}^{*} \mathcal{H}_{\mu \nu}^{\prime} \psi_{n \nu} .
\end{aligned}
$$

Repeating the steps outlined in the previous section, we find that the distribution of $Q_{X}$ is Gaussian with a width set by $Q_{\varepsilon}$,

$P\left(S, Q_{\varepsilon}, Q_{X}\right) \propto\left(\operatorname{det} Q_{\varepsilon}\right)^{-2 \beta N-3+3 \beta / 2} \exp \left[-\frac{\beta}{2} \operatorname{tr}\left(\tau_{\mathrm{H}} Q_{\varepsilon}^{-1}+\frac{1}{8}\left(\tau_{\mathrm{H}} Q_{\varepsilon}^{-1} Q_{X}\right)^{2}\right)\right]$.

The reason why the time-delay matrix $Q_{\varepsilon}$ sets the scale for the matrix $Q_{X}$ characterizing the response to the external parameter $X$ can be understood in a picture of classical trajectories [49]: for long delay times, the scattering properties are more sensitive to a perturbation of the system than for short delay times. Such an explanation in terms of classical trajectories is valid in the semiclassical limit $N \rightarrow \infty$ only. Our exact result (6.6) makes the relation between time delay and parameter response precise in the fully quantum mechanical regime of a small number of channels $N$.

The invariance property (6.3) and the Gaussian distribution (6.6) also hold if the scattering matrix depends on more than one external parameter. If the scattering matrix depends 
on parameters $X_{1}, \ldots, X_{n}$, the matrices $Q_{X},(j=1, \ldots, n)$ all have the same Gaussian distribution, with a width set by the symmetrized time-delay matrix $Q_{\varepsilon}$,

$$
\begin{aligned}
& P\left(S, Q_{\varepsilon}, Q_{X_{1}}, \ldots, Q_{X_{n}}\right) \propto\left(\operatorname{det} Q_{\varepsilon}\right)^{-N / 2-(\beta N+2-\beta)(n+2) / 2} \\
& \times \exp \left[-\frac{\beta}{2} \operatorname{tr}\left(\tau_{\mathrm{H}} Q_{\varepsilon}^{-1}+\frac{1}{8} \sum_{J=1}^{n}\left(\tau_{\mathrm{H}} Q_{\varepsilon}^{-1} Q_{X_{J}}\right)^{2}\right)\right] .
\end{aligned}
$$

\section{Conclusion}

In summary, we have presented a detailed derivation of the joint distribution $P\left(S, Q_{\varepsilon}\right)$ of the scattering matrix $S$ and the symmetrized time-delay matrix $Q_{\varepsilon}=-\mathrm{i} \hbar S^{-1 / 2}(\partial S / \partial \varepsilon) S^{-1 / 2}$ of a chaotic cavity with ideal leads. Our result, which was first reported in [17], reads

$$
P\left(S, Q_{\varepsilon}\right) \propto \theta\left(Q_{\varepsilon}\right) \operatorname{det}\left(Q_{\varepsilon}\right)^{-3 \beta N / 2-2+\beta} \exp \left(-\beta \tau_{\mathrm{H}} \operatorname{tr} Q_{\varepsilon}^{-1} / 2\right)
$$

where $\tau_{\mathrm{H}}=2 \pi \hbar / \Delta$ is the Heisenberg time and $\Delta$ is the mean level spacing in the cavity. The distribution $P\left(S, Q_{\varepsilon}\right)$ depends on the eigenvalues $\tau_{1}, \ldots, \tau_{N}$ of $Q_{\varepsilon}$ only; it does not depend on $S$, or on the eigenvectors of $Q_{\varepsilon}$. Our derivation was based on an old conjecture by Wigner [31], which we have proved in this paper, that the distribution functional $P[S(\varepsilon)$ ] of the ensemble of energy-dependent scattering matrices is invariant under unitary transformations. We generalized Wigner's conjecture to the dependence of $S$ on an external parameter $X$ and derived the distribution of parametric derivatives.

Throughout this paper we assumed ideal coupling of the chaotic cavity to the electron reservoirs. This requires ballistic point contacts. For the case of non-ideal coupling, i.e. if the point contacts contain a tunnel barrier, the distribution of the scattering matrix $S$ is given by the more general Poisson kernel instead of the circular ensemble [24,50]. For this situation, application of Wigner's conjecture is not correct and our method cannot be used to determine $P\left(S, Q_{\varepsilon}\right)$. A possibility to overcome this difficulty is to separate the direct and the fully ergodic scattering processes, by expressing the scattering matrix for non-ideal coupling as a composition of an $N \times N$ scattering matrix $S_{\text {erg }}$ of a fully ergodic and chaotic scatterer and a scattering matrix $S_{\mathrm{dr}}$ of dimension $2 N \times 2 N$ describing the direct scattering processes $[24,42,51]$,

$$
S=r+t^{\prime}\left(1-S_{\mathrm{erg}} r^{\prime}\right)^{-1} S_{\mathrm{erg}} t
$$

where the matrices $r, r^{\prime}, t$, and $t^{\prime}$ are $N \times N$ submatrices of $S_{\mathrm{drr}}$,

$$
S_{\mathrm{drr}}=\left(\begin{array}{cc}
r & t^{\prime} \\
t & r^{\prime}
\end{array}\right)
$$

The energy dependence of $S_{\mathrm{dIr}}$ can be neglected on energy scales corresponding to the invetse dwell time inside the cavity. Thus one obtains a relation between the statistical distributions of the time-delay matrices for the cases of non-ideal and ideal coupling. What remains is the cumbersome mathematical problem of performing the corresponding transformation of the different matrix variables. To our knowledge, no closed-form formula for the joint distribution of $S$ and $Q_{\varepsilon}$ is known, except for the case $N=1$ [51].

The supersymmetric approach used in $[4,12,13,33]$ is typically more general and systematic concerning the issue of non-ideal coupling, since there the particular symmetries of the scattering matrix ensemble in the ideal coupling case are not directly exploited. On the other hand, this technique is usually restricted to the computation of certain Green function averages only, and does not give access to the full distribution of the time-delay matrix. We believe that, in principle, in the supersymmetric approach it should be possible to determine averages 
of the form $\left\langle\operatorname{tr}\left(Q^{n}\right)\right\rangle$ for arbitrary $n$ (and hence the density of delay times) and for arbitrary coupling, without having to increase the dimension of the supermatrices involved. However, in order to obtain access to the full distribution of the matrix $Q_{\varepsilon}$, or at least to moments of the type $\left\langle(\operatorname{tr} Q)^{n}\right\rangle$, one has to increase the dimension of the supermatrices accordingly, which makes it apparently impossible to go beyond the case $n=2[4,12,13]$. It is amusing that the (now proven) half-a-century old conjecture of Wigner provides a route to the simple result (7 1) that seems unreachable by later supersymmetric technıques

\section{Acknowledgments}

This research was supported by the 'Nederlandse organisatie voor Wetenschappelijk Onderzoek' (NWO) and by the 'Stichting voor Fundamenteel Onderzoek der Materie' (FOM) PWB also acknowledges the support of the NSF under grants DMR 94-16910, DMR 96-30064, and DMR 97-14725

\section{References}

[1] Eisenbud L $1948 P h D$ Thests Princeton

[2] Wigner E P 1955 Phys Rev 98145

[3] Smith F T 1960 Phys Rev 118349

[4] Fyodorov Y V and Sommers H-J 1997 J Math Phys 381918

[5] Beenakker C W J 1997 Rev Mod Phys 69731

[6] Lyuboshits V L 1977 Phys Lett B 7241 Lyuboshits V L 1978 Yad Fiz 27948 (Engl transl 1978 Sov J Nucl Phys 27 502) Lyuboshts V L 1983 Yad Fiz 37292 (Engl transl 1978 Sov J Nucl Phys 37 174)

[7] Bauer M, Mello P A and McVoy K W 1979 Z Phys A 293151

[8] Lewenkopf C H and Weidenmuller H A 1991 Ann Phys NY 21253

[9] Harney H L, Dittes F-M and Muller A 1992 Ann Phys NY 220159

[10] Eckhardt B 1993 Chaos 3613

[11] Izrallev F, Saher D and Sokolov V V 1994 Phys Rev E 49130

[12] Lehmann N, Savin D V, Sokolov V V and Sommers H-J 1995 Physica D 86572

[13] Fyodorov Y V and Sommers H-J 1996 Phys Rev Lett 764709 Fyodorov Y V, Savin D V and Sommers H-J 1997 Phys Rev E 55 R4857

[14] Šeba P, Zyczkowskı K and Zakrewskı J 1996 Phys Rev E 542438

[15] Gopar V A, Mello P A and Buttiker M 1996 Phys Rev Lett 773005

[16] Brouwer P W and Buttuker M 1997 Europhys Lett 37441

[17] Brouwer P W, Frahm K M and Beenakker C W J 1997 Phys Rev Lett 784737

[18] Mucciolo E R, Jalabert R A and Pichard J L 1997 J Phystque 71267

[19] Comtet A and Texier C 1997 J Phys A Math Gen 308017

[20] Kouwenhoven L P, Marcus C M, McEuen P L, Tarucha S, Westervelt R M and Wingreen N S 1997 Mesoscopıc Election Transport (NATO ASI Series E345) ed L L Sohn et al (Dordrecht Kluwer)

[21] Smlansky U 1991 Chaos and Quantum Phys'cs ed M-J Giannom et al (Amsterdam North-Holland)

[22] Smlansky U 1995 Mesoscopic Quantum Physics ed E Akkermans et al (Amsterdam North-Holland)

[23] Blumel R and Smulansky U 1988 Phys Rev Lett 60477

Blumel R and Smilansky U 1990 Phys Rev Lett 64241

[24] Brouwer P W 1995 Phys Rev B 5116878

[25] Doron E, Smulansky U and Frenkel A 1990 Phys Rev Lett 653072

[26] Stem J, Stockmann H-J and Stoffregen U 1995 Phys Rev Lett 7553

[27] Kudrollı A, Kidambi V and Sridhar S 1995 Phys Rev Lett 75822

[28] Šeba P, Haake F, Kus M, Barth M, Kuhl U and Stockmann H-J 1997 Phys Rev E 562680

[29] Dyson F J 1962 J Math Phys 3140

[30] Mehta M L 1991 Random Matrices (New York Academıc)

[31] Wigner E P 1951 Ann Math $\mathbf{5 3} 36$

Wigner E P 1951 Proc Camb Phll Soc 47790

Wigner E P 1952 Ann Math 557 
[32] Brouwer P W 1997 PhD Thesis Leiden

[33] Verbaarschot J J M, Werdenmuller H A and Zurnbauer M R 1985 Phys Rep 129367

[34] Buttiker M and Chnsten T 1996 Quantum Transport in Semiconductor Submicron Structures (NATO ASI Seıes E326) ed B Kramer (Dordrecht Kluwer)

[35] van Langen S A, Silvestrov P G and Beenakker C W J 1998 Superlatt Microstruct 23691

[36] Brouwer P W, van Langen S A, Frahm K M, Buttıker M and Beenakker C W J 1997 Phys Rev Lett 79913

[37] Brouwer P W 1998 Phys Rev B 5810135

[38] Misirpashaev T Sh, Brouwer P W and Beenakker C W J 1997 Phys Rev Lett 791841

[39] Fyodorov Y V and Alhassid Y 1998 Phys Rev A 583375

[40] Guhr T, Muller-Groeling A and Weidenmuller H A 1998 Phys Rep 299189

[41] Hackenbroich G and Weidenmuller H A 1995 Phys Rev Lett 744118

[42] Friedman W A and Mello P A 1985 Ann Phys, NY 161276

[43] Slevin K and Nagao T 1994 Phys Rev B 502380

Nagao T and Slevin K 1993 J Math Phys 342075

Nagao T and Forrester P J 1995 Nucl Phys B 435401

[44] Brézin E, Hıkamı S and Zee A 1996 Nucl Phys B 464411

[45] Edelman A 1988 SIAM J Matrix Anal Appl 9543

[46] Baker T H, Forrester P J and Pearce P A J Phys A Math Gen 316087

[47] Abramowitz M and Stegun I A 1964 Handbook of Mathematical Functions (Washington, DC National Bureau of Standards) ch 22

[48] Brouwer P W and Beenakker C W J 1996 Phys Rev B 5412705

[49] Jalabert R A, Baranger H U and Stone A D 1990 Phys Rev Lett 652442

[50] Mello P A, Pereyra P and Seligman T H 1985 Ann Phys, NY 161254

[51] Gopar V A and Mello P A 1998 Europhys Lett 42131 